\title{
The cannabinoid WIN55,212-2 abrogates dermal fibrosis in scleroderma bleomycin model
}

${ }^{1}$ Rheumatology Unit, Department of Clinical Medicine and Immunological Sciences, University of Siena, Siena, Italy ${ }^{2}$ Department of Internal Medicine 3, University of Erlangen-Nuremberg, Germany ${ }^{3}$ Department of

Pathophysiology, Experimental Medicine and Public Health, University of Siena, Siena, Italy

\section{Correspondence to}

Enrico Selvi, Rheumatology Unit, Department of Clinical Medicine and Immunological Sciences, University of Siena, Viale Bracci 1, 53100 Siena, Italy; enrico.selvi@gmail.com

Accepted 1 November 2010 Published Online First 21 December 2010

\author{
Epifania Balistreri, ${ }^{1}$ Estrella Garcia-Gonzalez, ${ }^{1}$ Enrico Selvi, ${ }^{1}$ Alfiya Akhmetshina, ${ }^{2}$ \\ Katrin Palumbo, ${ }^{2}$ Sauro Lorenzini, ${ }^{1}$ Roberta Maggio, ${ }^{1}$ Monica Lucattelli, ${ }^{3}$ \\ Mauro Galeazzi, ${ }^{1}$ Jörg W H Distler ${ }^{2}$
}

\begin{abstract}
Objectives There is increasing evidence that the endocannabinoid system may be involved in pathological fibrosis, and that its modulation might limit fibrotic responses. The aim of this study was to examine the capacity of a synthetic cannabinoid receptor agonist to modify skin fibrosis in the bleomycin mouse model of scleroderma.
\end{abstract}

Methods Skin fibrosis was induced by local injections of bleomycin in two groups of DBA/2J mice. One group was cotreated with the synthetic cannabinoid WIN55,212-2 at $1 \mathrm{mg} / \mathrm{kg} /$ day. Skin fibrosis was evaluated by histology and skin thickness and hydroxyproline content were quantified. Markers of fibroblast activation, including $\alpha$ smooth muscle actin and the profibrotic cytokines transforming growth factor (TGF) $\beta$, connective tissue growth factor (CTGF) and platelet-derived growth factor (PDGF)-BB, were examined. Levels of PSMAD2/3, which are crucial in extracellular matrix overproduction, were analysed.

Results Bleomycin treatment induced typical skin fibrosis. Upon WIN55,212-2 treatment dermal fibrosis was completely prevented. Subcutaneous inflammatory cell infiltration, dermal thickness and collagen content resulted similar to those of the control group. The synthetic cannabinoid prevented fibroblasts activation induced by bleomycin, paralleled by a strong inhibition of TGFB, CTGF and PDGF-BB expression. Phosphorylation of SMAD2/3 was significantly downregulated after WIN55,212-2 exposure.

Conclusions Taken together, the results indicate that the synthetic cannabinoid WIN55,212-2 is capable of preventing skin fibrosis in a mouse model of scleroderma.

\section{INTRODUCTION}

Systemic sclerosis (SSc) is a connective tissue disease of unknown aetiology that affects the skin and internal organs. In early stages, vascular injury and inflammation lead to the fibrosis of tissues, resulting in irreversible damage. ${ }^{1} 2$ The inflammatory early stage of the disease seems to be necessary in order to activate fibroblasts to overproduce extracellular matrix components (ECM). ${ }^{1}$ Transforming growth factor (TGF) $\beta$ signalling plays a key role in the pathogenesis of SSc fibrosis by regulating multiple cellular functions, including the inhibition and stimulation of cell growth, cell death and cellular differentiation. ${ }^{3-5}$ TGF $\beta$ signalling occurs predominantly by phosphorylation of cytoplasmic proteins belonging to the Smad family. ${ }^{6}$ In SSc, dysregulated TGF $3 / \mathrm{SMAD} 2 / 3$ signalling is thought to be crucial in the transcriptional upregulation of type I collagen. ${ }^{7}$ However, in addition to the canonical TGF $\beta$ pathway, several other pathways contribute to the aberrant activation of fibroblasts in SSc. ${ }^{1}$

It has been recently suggested that the endocannabinoid system could be upregulated in pathological fibrosis, and that its modulation might limit the progression of uncontrolled fibrogenesis. ${ }^{8}$ The endocannabinoid system comprises the two specific cannabinoid receptors (CB1 and CB2), their endogenous ligands and the machinery dedicated to endocannabinoid synthesis and degradation. ${ }^{9}$ Cannabinoids may behave as profibrotic or antifibrotic agents, depending on their interaction with CB1 or CB2 receptors. ${ }^{10}$ Furthermore, an antifibrotic effect of cannabinoids has been described independently from CB1/CB2-receptor activation. ${ }^{11-14}$ These data point towards a potential use of cannabinoids to limit or even reverse tissue damage in pathological fibrosis. However, the exact mechanism of action and its dependence on the cannabinoid receptors remains to be determined.

On this basis, we performed the present study in order to verify whether WIN55,212-2 could modify skin fibrosis in a mouse model of bleomycininduced scleroderma.

\section{MATERIALS AND METHODS \\ Animals and experimental protocol}

Skin fibrosis was induced in two groups of 6-weekold DBA/2J mice by local injections of bleomycin (BLM) for 21 days. Briefly, $100 \mu \mathrm{l}$ of BLM dissolved in $0.9 \% \mathrm{NaCl}$ at a concentration of $0.5 \mathrm{mg} / \mathrm{ml}$ were administered every other day by subcutaneous injection into a defined area of $1 \mathrm{~cm}^{2}$ on the upper back. To evaluate the effect of the synthetic cannabinoid, a group of BLM-challenged mice was treated with WIN55,212-2 (Tocris, Missouri, USA). WIN55,212-2 was dissolved in phosphate-buffered saline/0.3\% Tween80/dimethylsulfoxide (DMSO) at a concentration of $1 \mathrm{mg} / \mathrm{kg} /$ day in a total volume of $100 \mu \mathrm{l}$, and administered by subcutaneous injections, in the same area as the BLM treatment, for 21 days. Control mice were injected with equal volumes of solvent to control for potential effects of DMSO. Treatment with WIN55,212-2 was started in parallel to bleomycin challenge. Each treatment group consisted of seven mice. After 21 days all the animals were killed by cervical dislocation, then the injected skin was removed and processed for analysis. The local ethical committee approved all animal experiments. 


\section{Histological analysis}

Three lesional skin samples were taken from every animal of each group and $5 \mu \mathrm{m}$ skin sections (three for each skin sample) were stained with haematoxylin and eosin. Dermal thickness was calculated at $10 \times$ microscopic magnification by measuring the distance between the dermal-epidermal junction and the dermal-subcutaneous fat junction $(\mu \mathrm{m})$ in five randomly selected fields for each skin section. Two different examiners performed the evaluation blindly.

\section{Determination of the hydroxyproline content}

The collagen content of lesional skin was evaluated by colourimetric quantification of hydroxyproline on three different skin biopsies ( $3 \mathrm{~mm}$ punch) taken from every animal in each group. ${ }^{15}$ Absorbencies were measured at $560 \mathrm{~nm}$ in triplicate. The results were expressed as $\mu \mathrm{g}$ of hydroxyproline per biopsy (mean \pm SEM).

\section{Immunohistochemistry and immunofluorescence staining}

Skin sections $(5 \mu \mathrm{m})$ were processed in order to evaluate the expression of $\alpha$ smooth muscle actin ( $\alpha$-SMA) (mouse monoclonal antibody) (Santa Cruz Biotechnology, Santa Cruz, California, USA) TGF $\beta$ (mouse monoclonal antibody), connective tissue growth factor (CTGF) (rabbit polyclonal antibody) and plateletderived growth factor (PDGF)-BB (rabbit polyclonal antibody) (Abcam, Cambridge, Massachusetts, USA). Positive cells were visualised by diaminobenzidine (DAB), using a peroxidase substrate kit (Dako, Italy). Cell counts were performed blindly by two different operators, at 40× magnification, in five non-contiguous microscope fields of three sections from each lesional skin sample. Results are expressed as mean \pm SEM of positive spindleshaped fibroblastic cells per field. PSMAD2/3 positive cells were detected by immunofluorescence technique by incubating $5 \mu \mathrm{m}$ skin sections with anti-PSMAD2/3 (goat polyclonal antibody) (Santa Cruz Biotechnology).

\section{Western blot analysis}

Protein samples obtained from lesional skin samples from every mouse in each group were used for western blot analysis. Aliquots of protein were probed for SMAD2/3 (rabbit polyclonal antibody), PSMAD2/3 (goat polyclonal antibody) (Santa Cruz Biotechnology). For confirmation of equal loading of proteins, the amount of $\beta$-tubulin was determined using mouse anti- $\beta$-tubulin antibody (Sigma, Italy). Results are expressed as mean \pm SEM of three different assays.

\section{Statistical analysis}

All values presented are expressed as the mean \pm SEM. Analysis of variance was used to compare multiple means, followed by the Bonferroni post hoc test. A statistical significance level of $95 \%(p<0.05)$ was applied. All data analyses were performed using the Sigma Stat V.3.5 (Sigma Stat, Ashburn, Virginia, USA) statistical software package.

\section{RESULTS \\ WIN55,212-2 prevented bleomycin-induced dermal fibrosis}

To evaluate the antifibrotic effect of WIN55,212-2, BLMchallenged mice were treated with WIN55,212-2 at the concentration of $1 \mathrm{mg} / \mathrm{kg} /$ day for 21 days. Skin fibrosis was quantified by determining the dermal thickness and the hydroxyproline content of lesional skin. BLM treatment resulted in a considerable increase in dermal thickness compared to controls (BLM: $393 \pm 16 \mu \mathrm{m}$ vs control: $204 \pm 8 \mu \mathrm{m} ; \mathrm{p}<0.001)($ mean \pm SEM). Upon administration of WIN55,212-2, BLM-induced dermal thickening was completely abrogated (BLM/WIN55,212-2: 197 $\pm 11 \mu \mathrm{m} ; \mathrm{p}<0.001)$ (figure 1A). Beyond dermal thickening, lesional skin from BLM-challenged mice revealed the typical pathological features of SSc at histological examination, including atrophy of the subcutaneous fat tissue and an accumulation of inflammatory cells in the deeper dermal layers and perivascular spaces. WIN55,212-2 treatment partially
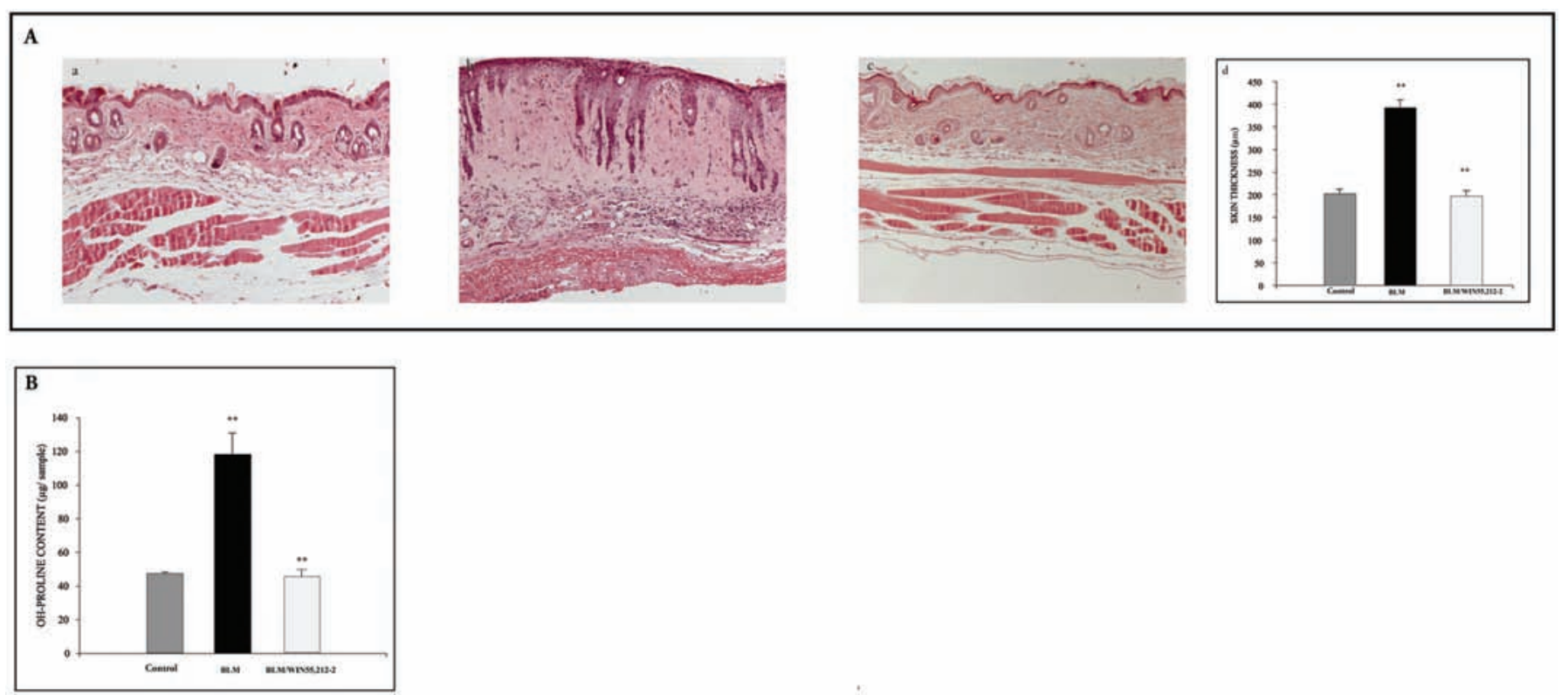

Figure 1 The synthetic cannabinoid WIN55,212-2 prevented bleomycin-induced dermal fibrosis. (A) (a) Control, (b) bleomycin (BLM), (c) BLM/ WIN55,212-2. In BLM-challenged mice dermal thickening, epidermal hypertrophy, subcutaneous fat atrophy and accumulation of mononuclear and polymorphonuclear leucocytes in the deeper dermal layers and perivascular spaces were observed. Upon WIN55,212-2 treatment, subcutaneous inflammatory infiltration and dermal fibrosis were consistently reduced and epidermal and subcutaneous adipose layers were preserved. Haematoxylin and eosin staining, original magnification $10 \times$. (d) Histograms showing dermal thickness quantification $(\mu \mathrm{m}) .{ }^{* *} p<0.001$. All the results are expressed as mean \pm SEM. (B) Collagen content in $3 \mathrm{~mm}$ punch biopsy of skin as measured by quantification of hydroxyproline ( $\mu \mathrm{g} / \mathrm{sample})$. ${ }^{* *} p<0.001$. All the results are expressed as mean \pm SEM. 

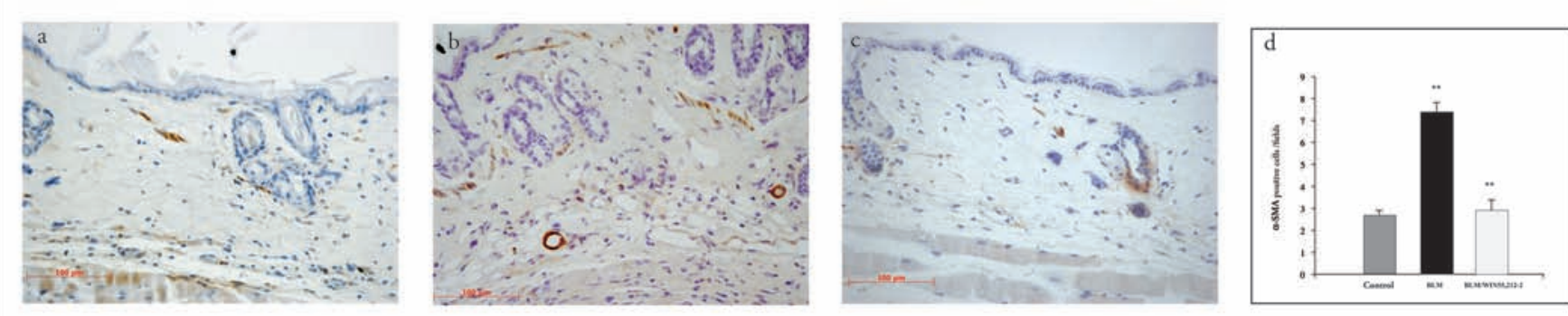

Figure 2 The synthetic cannabinoid WIN55,212-2 inhibit fibroblast activation. (A) Control, (B) bleomycin (BLM), (C) BLM/WIN55,212-2. Immunostain showing a strong downregulation of $\alpha$-smooth muscle actin (SMA). Original magnification $20 \times$. (D) $\alpha$-SMA positive spindle-shaped fibroblastic cells per microscopic field. ${ }^{* *} \mathrm{p}<0.001$. All the results are expressed as mean \pm SEM.

preserved the subcutaneous adipose layer and strongly reduced subcutaneous infiltration by inflammatory cells (figure 1A). The histological evidence of dermal fibrosis in BLM-challenged mice was associated with a twofold increase in the hydroxyproline content compared to controls (BLM: $118 \pm 13 \mu \mathrm{g} / \mathrm{sample}$ vs control: $47 \pm 1 \mu \mathrm{g} /$ sample; $\mathrm{p}<0.001)$ (mean \pm SEM). Treatment with WIN55,212-2 completely abrogated the BLM-induced collagen deposition, as levels of hydroxyproline in the cotreated group were equal to those in the control group (BLM/WIN55,212-2: $44 \pm 4 \mu \mathrm{g} /$ sample; $\mathrm{p}<0.001)($ mean \pm SEM) (figure 1B).

\section{WIN55,212-2 inhibited fibroblast activation}

Myofibroblasts are specialised fibroblasts characterised by the cytoskeletal protein $\alpha$-SMA that are a major source of ECM and profibrotic cytokines in fibrotic diseases. After BLM challenge there was a strong increase in the number of $\alpha$-SMA positive fibroblastic cells in lesional skin compared to controls (BLM: $7.4 \pm 0.4$ vs control: $2.7 \pm 0.2 ; \mathrm{p}<0.001)($ mean \pm SEM). Treatment with WIN55,212-2 resulted in a mean \pm SEM of $2.0 \pm 0.5$ of $\alpha$-SMA positive spindle-shaped cells per field compared to BLM group $(p<0.001)$, indicating that the synthetic cannabinoid prevented the myofibroblast differentiation induced by BLM (figure 2).

\section{WIN55,212-2 inhibited PDGF and TGF $\beta$ signalling}

The profibrotic cytokine PDGF plays a key role in fibroblast activation and has been implicated in the transdifferentiation of pericytes into myofibroblasts. Lesional skin from BLM-treated mice showed a marked increase in the number of PDGF-BB positive spindle-shaped fibroblastic cells per field compared to controls (BLM: $16.7 \pm 0.9$ vs control: $5.9 \pm 0.5 ; \mathrm{p}<0.001)($ mean $\pm S E M)$. Treatment with WIN55,212-2 significantly downregulated the number of PDGF-BB positive cells (BLM/WIN55,212-2: 6.8 \pm 0.7 ; $\mathrm{p}<0.001$ ) (mean \pm SEM) (figure $3 \mathrm{~A}$ ).

TGF $\beta$ promotes early fibrotic response in scleroderma, which is subsequently maintained by CTGF. Lesional skin from BLMchallenged mice showed a marked increase of TGF $\beta$ (BLM: $6.42 \pm 0.48$ vs control: $0.93 \pm 0.33 ; \mathrm{p}<0.05$ ) and CTGF (BLM: $4.25 \pm 0.29$ vs control: $0.92 \pm 0.16 ; \mathrm{p}<0.05)$ positive spindle-shaped fibroblastic cells per field compared to controls. WIN55,212-2 resulted in a downregulation of TGF $\beta$ (BLM/WIN55,212-2: $2.84 \pm 0.26$ vs BLM: $6.42 \pm 0.48 ; \mathrm{p}<0.05)$ and CTGF expression (BLM/WIN55,212-2: $2.67 \pm 0.44$ vs BLM: $4.25 \pm 0.29 ; \mathrm{p}<0.05$ ) (mean \pm SEM) (figure $3 B, C$ ).

SMAD proteins are the downstream mediators of canonical TGF 3 signalling. Mild PSMAD2/3 phosphorylation was observed in skin samples from controls, while the BLM challenge induced a robust positivity for $\mathrm{PSMAD} 2 / 3$ in lesional skin. Upon WIN55,212-2 treatment a significant inhibition of
PSMAD2/3 was observed, achieving similar levels to the control samples (figure $3 \mathrm{D}$ ). Accordingly, western blot analysis showed a twofold decrease in PSMAD2 and PSMAD3 protein levels in the BLM/WIN55,212-2 group compared to the BLM-challenged mice $(p<0.05)($ mean \pm SEM) (figure $3 E)$.

\section{DISCUSSION}

The synthetic cannabinoid WIN55,212-2 markedly attenuated the dermal fibrosis and ECM deposition in bleomycin induced dermal fibrosis. The inhibition of skin thickening was accompanied by the downregulation of myofibroblast transdifferentiation and by a parallel inhibition of the profibrotic cytokines TGF $\beta$, CTGF and PDGF-BB. These cytokines are reciprocally upregulated in an autocrine/paracrine mechanism that leads to the constitutive activation of fibrogenesis in SSc. ${ }^{16-19}$ The finding that the dysregulated TGF $\beta$, CTGF and PDGF expression in bleomycin-treated mice was inhibited upon WIN55,212-2 exposure may suggest a role of the cannabinoid in disrupting the selfperpetuating loop that occurs in aberrant fibroblast activation and ECM deposition.

Bleomycin-induced dermal fibrosis is considered a reliable experimental model for scleroderma studies as it reflects early stages of SSc with increased release of collagen and other ECM components, migration of inflammatory cells into the skin and substitution of the adipose layer by fibrotic tissue. ${ }^{18}$ Our results showed a significant decrease in inflammatory cells infiltrating the subcutis of BLM-challenged mice upon WIN55,212-2 treatment. It could therefore be assumed that the antifibrotic effect of WIN55,212-2 in our study might be due to the antiinflammatory activity of cannabinoids. ${ }^{12}$ However, we suggest that the inhibition of dermal fibrosis induced by WIN55,212-2 goes beyond a mere anti-inflammatory activity. ${ }^{13}$ Cultured SSc fibroblasts serve as experimental model in which it is possible to study the mechanisms involved in ECM overproduction independently of other actors that orchestrate the early inflammatory phase of the scleroderma scenario. In this setting, WIN55,212-2 exerted potent antifibrotic effects on SSc fibroblasts that were not mediated by the classical cannabinoid receptors $\mathrm{CB} 1 / \mathrm{CB} 2$ and cultured SSc fibroblasts did not alter the production of ECM upon CB2-receptor modulation. ${ }^{12}{ }^{14}$ Taken together, these data suggest that cannabinoids exert an antifibrotic as well as anti-inflammatory effect, although the role of the endocannabinoid system in the pathogenesis of fibrosis remains to be fully elucidated. Although these effects highlight direct antifibrotic effects of WIN55,212-2 on fibroblasts, we cannot exclude that effects on other cell types might have also contributed to the potent antifibrotic effects of WIN55,212-2 in bleomycin induced dermal fibrosis. 

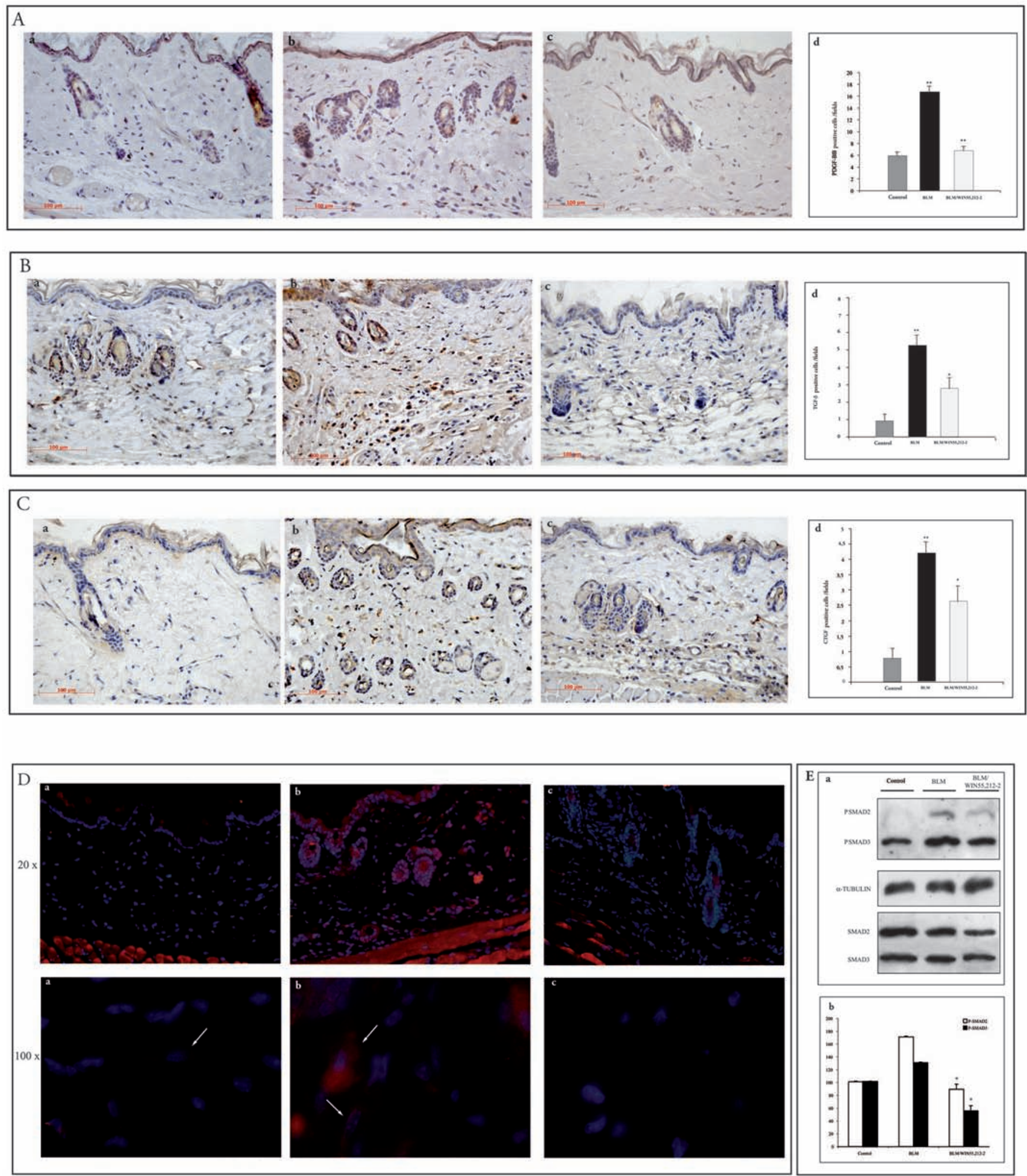

Figure 3 The synthetic cannabinoid WIN55,212-2 inhibited platelet-derived growth factor (PDGF) and transforming growth factor (TGF) $\beta$ signalling. (a) Control, (b) bleomycin (BLM), (c) BLM/WIN55,212-2. Immunostain showing a strong downregulation of PDGF-BB (A), TGF $\beta$ (B) and connective tissue growth factor (CTGF) (C) expression. Original magnification 20×. (d) PDGF-BB (A), TGF $\beta$ (B) and CTGF (C) positive spindle-shaped fibroblastic cells per microscopic field. ${ }^{*} p<0.05,{ }^{* *} p<0.001$. (D) Immunofluorescence showing SMAD2/3 phosphorylation (in red). Nuclei stained in blue by 4 ',6diamidino-2-phenylindole (DAPI). Upon WIN55,212-2 treatment PSMAD2/3 immunoreactivity was markedly reduced compared to BLM-treated mice. Original magnifications $20 \times$ and $100 \times$. (E) Western blot analysis of PSMAD2 and PSMAD3 (a) and its densitometric analysis (b) showing inhibition of SMAD2 and SMAD3 phosphorylated proteins after WIN55,212-2 treatment. All the results are expressed as mean \pm SEM. ${ }^{*} p<0.05$. 
In conclusion, we propose that WIN55,212-2 is able to disrupt dermal fibrogenesis in a model of bleomycin-induced SSc through a dual anti-inflammatory and antifibrotic mechanism, by promoting downregulation of the overamplified PDGF and TGF $\beta$ signalling pathways. Further studies are warranted in order to confirm cannabinoids as antifibrotic agents in models of later stages of SSc and to investigate whether cannabinoids can also induce regression of pre-established fibrosis.

Competing interests None.

Provenance and peer review Not commissioned; externally peer reviewed.

\section{REFERENCES}

1. Varga J, Abraham D. Systemic sclerosis: a prototypic multisystem fibrotic disorder. $J$ Clin Invest 2007;117:557-67.

2. Abraham DJ, Eckes B, Rajkumar V, et al. New developments in fibroblast and myofibroblast biology: implications for fibrosis and scleroderma. Curr Rheumatol Rep 2007:9:136-43.

3. Ihn H. The role of TGF-beta signaling in the pathogenesis of fibrosis in scleroderma. Arch Immunol Ther Exp (Warsz) 2002;50:325-31.

4. Leask A, Abraham DJ. TGF-beta signaling and the fibrotic response. FASEB J 2004;18:816-27.

5. Jinnin M. Mechanisms of skin fibrosis in systemic sclerosis. J Dermatol 2010;37:11-25

6. Kretzschmar M, Massagué J. SMADs: mediators and regulators of TGF-beta signaling. Curr Opin Genet Dev 1998;8:103-11.
7. Mori Y, Chen SJ, Varga J. Expression and regulation of intracellular SMAD signaling in scleroderma skin fibroblasts. Arthritis Rheum 2003;48:1964-78.

8. Teixeira-Clerc F, Julien B, Grenard P, et al. The endocannabinoid system as a novel target for the treatment of liver fibrosis. Pathol Biol 2008;56:36-8.

9. Demuth DG, Molleman A. Cannabinoid signalling. Life Sci 2006;78:549-63.

10. Parfieniuk A, Flisiak R. Role of cannabinoids in chronic liver diseases. World J Gastroenterol 2008;14:6109-14.

11. Michalski CW, Maier M, Erkan M, et al. Cannabinoids reduce markers of inflammation and fibrosis in pancreatic stellate cells. PLOS ONE 2008;3:e1701.

12. Akhmetshina A, Dees $\mathrm{C}$, Busch N, et al. The cannabinoid receptor CB2 exerts antifibrotic effects in experimental dermal fibrosis. Arthritis Rheum 2009;60:1129-36.

13. Servettaz A, Kavian N, Nicco C, et al. Targeting the cannabinoid pathway limits the development of fibrosis and autoimmunity in a mouse model of systemic sclerosis. Am J Pathol 2010;177:187-96.

14. Garcia-Gonzalez E, Selvi E, Balistreri E, et al. Cannabinoids inhibit fibrogenesis in diffuse systemic sclerosis fibroblasts. Rheumatology (Oxford) 2009;48:1050-6.

15. Kivirikko KI, Ganser V, Engel J, et al. Comparison of poly-L-proline I and II as inhibitors of protocollagen hydroxylase. Hoppe-Seyler's Z Physiol Chem 1967; 348:1341-4.

16. Trojanowska M. Role of PDGF in fibrotic diseases and systemic sclerosis. Rheumatology (Oxford) 2008;47(Suppl 5):v2-4.

17. Yamamoto T. The bleomycin-induced scleroderma model: what have we learned for scleroderma pathogenesis? Arch Dermatol Res 2006;297:333-44.

18. Yamamoto T. Animal model of systemic sclerosis. J Dermatol 2010;37:26-41.

19. Lakos G, Takagawa S, Chen SJ, et al. Targeted disruption of TGF-beta/Smad3 signaling modulates skin fibrosis in a mouse model of scleroderma. Am J Pathol 2004;165:203-17. 


\section{A The cannabinoid WIN55, 212-2 abrogates dermal fibrosis in scleroderma bleomycin model}

Epifania Balistreri, Estrella Garcia-Gonzalez, Enrico Selvi, Alfiya Akhmetshina, Katrin Palumbo, Sauro Lorenzini, Roberta Maggio, Monica Lucattelli, Mauro Galeazzi and Jörg W H Distler

Ann Rheum Dis 2011 70: 695-699 originally published online December 21,2010

doi: 10.1136/ard.2010.137539

Updated information and services can be found at:

http://ard.bmj.com/content/70/4/695

\section{These include:}

References This article cites 19 articles, 3 of which you can access for free at: http://ard.bmj.com/content/70/4/695\#BIBL

Email alerting service

Receive free email alerts when new articles cite this article. Sign up in the box at the top right corner of the online article.

\section{Notes}

To request permissions go to:

http://group.bmj.com/group/rights-licensing/permissions

To order reprints go to:

http://journals.bmj.com/cgi/reprintform

To subscribe to BMJ go to:

http://group.bmj.com/subscribe/ 